\title{
Diffuse large B cell lymphoma of the oral cavity - Case report
}

\author{
Anna Gawęda ${ }^{1,2}$, Ewa Jach ${ }^{1,2}$, Jolanta Wojciechowicz ${ }^{1,2}$, Bożena Sokołowska ${ }^{1,2}$, Tomasz Tomaszewski ${ }^{1,2}$ \\ ${ }^{1}$ Department of Maxillofacial Surgery, Medical University, Lublin, Poland \\ ${ }^{2}$ Haematooncology Department, Medical University, Lublin, Poland
}

Gawęda A, Jach E, Wojciechowicz J, Sokołowska B, Tomaszewski T. Diffuse large B cell lymphoma of the oral cavity - Case report. J Pre-Clin Clin Res. 2014; 8(1): 27-29.

\section{Abstract}

The study presents an atypical location of Non-Hodgkin lymphoma of the oral cavity in the area of the palate. Pathological changes of the mucosa of the palate in an 85-year-old female patient had been developing for many years as a result of upper denture irritation without any major symptoms. Histological examination revealed a Diffuse large B cell lymphoma. Due to being advanced in years, the patient was qualified for the local radiotherapy. In this particular case, the clinical picture of lymphoma imitated an inflammation of the mucosa of the oral cavity which resulted in delayed diagnosis and treatment.

\section{Key words}

diffuse large B cell lymphoma, oral cavity, palate, ulceration

\section{INTRODUCTION}

Non-Hodgkin lymphoma (NHL) is a group of histologically and biologically heterogeneous clonal malignant diseases arising from the lymphoid system. NHL constitute $4-5 \%$ of all cancers and are the sixth cause of death in this group $[1,2,3]$. In Europe and the USA, 85\% of lymphomas are the B-cell type. They affect mainly lymph nodes, and the lymphatic organs (liver, spleen), but they can also occur primarily extra-nodularly. In the area of the head and neck they are located mainly in salivary glands, eyeballs, the nasopharynx $x_{2}$ maxillary sinuses, and in some rare cases at the bottom of the oral cavity and lips $[4,2,5]$. Extranodular lymphoma are located often in gastrointestinal tract, primarily in the stomach $[2,4]$.

Primary oral lymphomas account for less than 5\% of oral malignant tumours and for about $1 \%$ of lymphomas as a whole [6].

Non-Hodgkin lymphomas are classified using the World Health Organization (WHO) system based on the immunological and molecular characteristics of malignant lymphocytes [7].

The main method of treatment of lymphomas, regardless of their primary location, is chemotherapy and radiotherapy $[8$, $4,2]$. Surgery is performed only for diagnostic purposes since it provides with material used in histological examination [4]. Occasionally, some in-situ and extra-nodal initial lesions are removed surgically (for example, from the thyroid, spleen) [9]. Varied clinical picture of lymphomas imitating inflammation often lead to misdiagnosis and consequently incorrect treatment. Thus, correct and instant implementation of chemo- or radiotherapy becomes the major factor which determines the patient's later fate.

Address for correspondence: Ewa Gawęda, Department of Maxillofacial Surgery Medical University, Staszica 11, 20-081 Lublin, Poland e-mail: agaweda5@wp.pl

Received: 19 February 2014; accepted: 13 May 2014

\section{CASE REPORT}

A female aged 85 was referred to the Maxillofacial Surgery Department at the Medical University in Lublin by a prosthodontist with whom she had made an appointment to have her new complete upper denture made. Lesions of the palate mucosa had made the planned prosthetic procedure impossible. The lesion had been growing slowly and painlessly for several years. Some local anti-inflammatory treatment did not bring the expected therapeutic effect. The medical history of the patient included hypothyroidism and cataract surgery performed a year before.

On admission, the physical findings showed two ulcerations on the soft and hard palate mucosa with white and yellow coating which were moderately painful and bled after being touched. The larger lesion which measured approx. $1 \mathrm{~cm}$ in diameter was located on the left side of the soft palate and extended into the oedematous, inflammatory lesion on the palatoglossal arch (Fig. 1, 2). The examination revealed bilateral, painful, moderately enlarged submandibular lymph nodes shifting to the skin and bottom of the nasal cavity. Surgical biopsy of the lesion was performed under $2 \%$ lignocaine local anaesthesia. Histological examination (No. 73462) showed a malignant neoplasm, presumably a lymphoma. Further immunohistochemical diagnosis determined a Diffuse large B-cell lymphoma (DLBCLNOS-not otherwise specified) - LCA(+), CD20(+), BCL2(+), BCL6(+), MUM1(+), CD10(-), CD5(-), KI67(+++), labelling index $-90 \%$. Among additional examinations, a blood test was carried out to determine the level of TSH, FT3, FT4; they were within normal limits. A dental panoramic radiograph and computer tomography examination of the facial skeleton were carried out. The latter revealed thickening of the soft tissue of the palate and the bottom of the nasal cavity, hypertrophy of the inferior nasal concha and the mucosa of the nasal septum. The bone tissue of the hard palate and inferior nasal concha thinned with the occurrence of density reduction in the bone structure, pathological infiltration in soft tissues of the lower nasal septum and both nasal passages extending into the alveolar recess of the right maxillary sinus (Fig. 3). Trephine biopsy was not ordered due to the 


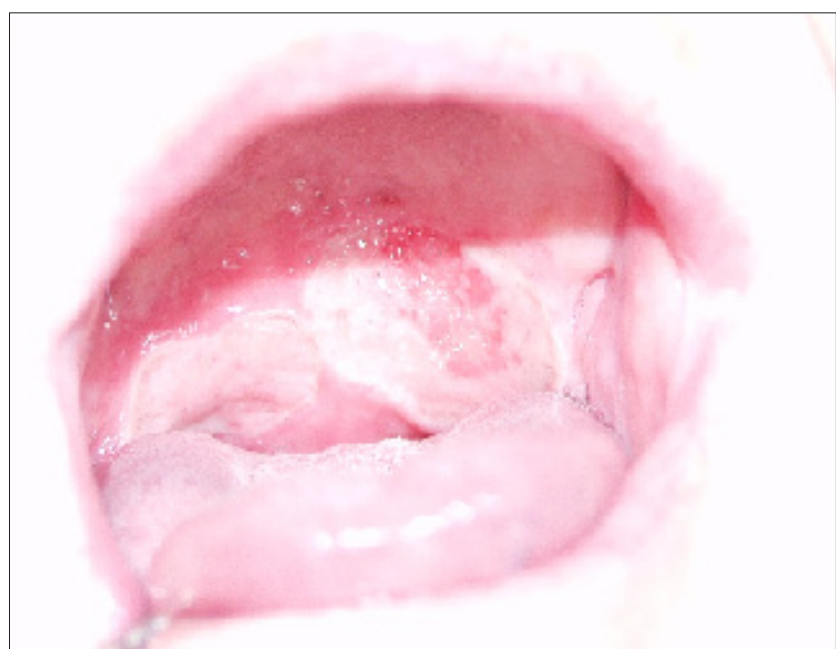

Figure 1. Ulceration on the soft and hard palate

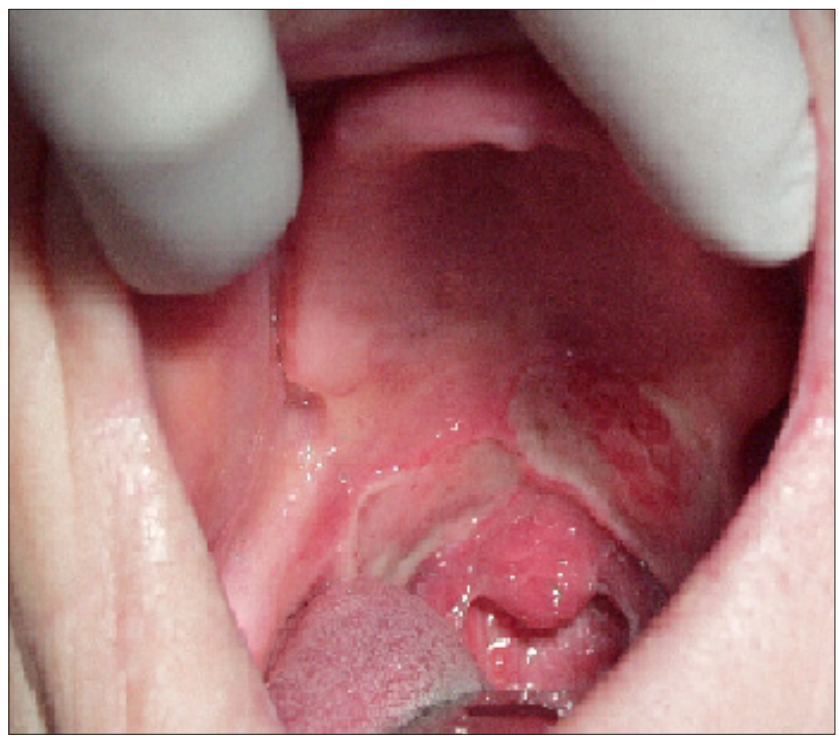

Figure 2. Lymphoma affecting the soft palate, edge of the hard palate and right palatoglossal arch

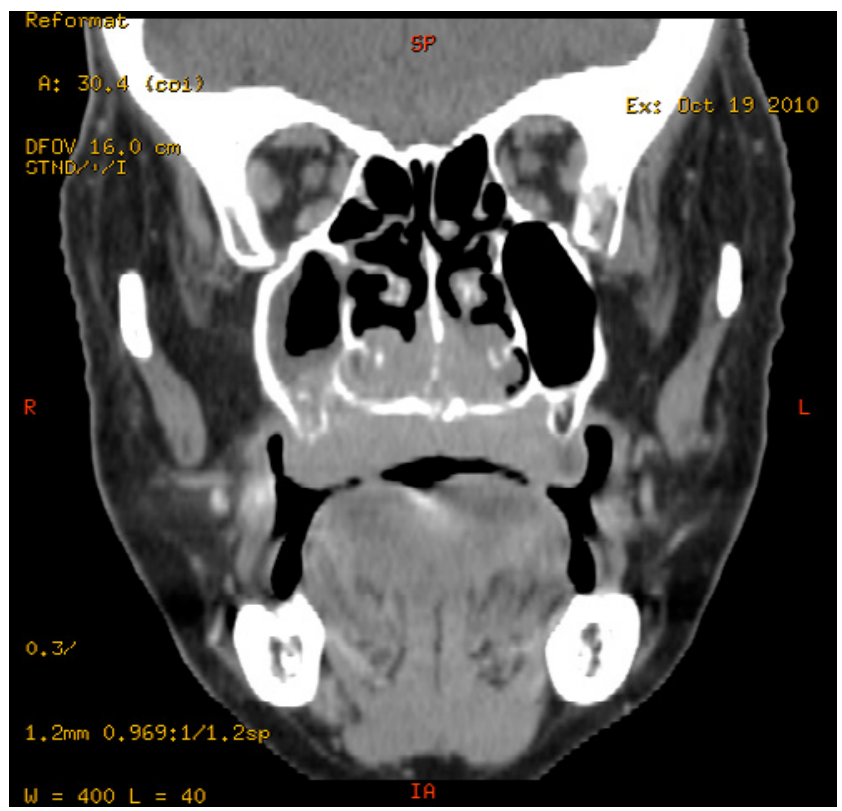

Figure 3. CT scan - lesions in the right maxillary bone sinus in lower part of the nasal septum and in the alveolar process of right maxilla patient's age and scarce usefulness of the examination in further treatment. The patient was referred to the St. Johns' Oncology Centre in Lublin to undergo local radiotherapy of the facial skeleton.

\section{DISCUSSION}

Primary oral lymphoma most commonly presents as a painless local mass that gradually increases in size with superficial ulceration. Clinical signs and symptoms of lymphoma of the paranasal sinuses include a mass in the nasal cavity, facial pain, paresthesia, sinusitis, nasal discharge, oedematous eyelids and proptosis of orbital invasion $[5,4]$. The case was solely manifested by an ulceration. Limited-stage primary non-tonsillar oral Diffuse large B-cell lymphoma (DLBCL) is rare in non-HIV patients. Only 12 cases have been reported to date [10]. DLBCL may occur at all ages, but generally in patients more than 40 years of age. The median age of the patient in the presented case was 65 years (57-77years), and females accounted for $64 \%$ of the total [10]. In $50 \%$ of patients, DLBCL presents with localized stage. DLBCL is characterized by a diffuse pattern of proliferation of the relatively large cells. There are many histological subtypes of DLBCLs. Among them, DLBCL-NOS (not otherwise specified) subtype is characterized by pan- B markers (CD20+, CD 79a+), and a Ki67 labeling index less than 90\% (Ki67 - more than 99\% is typical for Burkitt lymphoma) [11]. Plasmablastic subtype of DLBCL is also a rare tumor, but it often occurs in the mouth, and is most common in human immunodeficiency virus (HIV)-positive males [12]. As a part of the diagnostic examinations a biopsy of the lymph node or extra-nodal mass, as well as imaging examinations (USG, CT, MR), are required. Histological diagnosis should be carried out by an expert haematopathologist. Extra-nodal DLBCLs, despite their subtypes, usually have a good prognosis. The percentage of 5 -year survival is as high as $80 \%[5,13]$.

The literature presents some rare cases of extra-nodal lymphoma localized on the palate. A case of DLBCL in a 60 -year-old male patient was manifested by a slightly painful ulcerated growth on the right maxillary alveolar ridge extending onto the palate. The patient was referred to the oncology department, where chemotherapy was administered. Unfortunately, he died after two cycles of the treatment [5]. Frei [3] presents a case of non-Hodgkin lymphoma in a 76-year-old male. There was an exophytic lesion in the region of the right hard and soft palate. Radiographic examination showed a massive thickening of the right sinus membrane and erosion of the lateral and basel cortical sinus walls in the right maxilla. Chung-Che Chang [14] illustrates a case of mantle cell lymphoma (MTL) atypically located on the hard palate. A more typical location for this type of lymphoma is the nasopharynx and Waldeyer's tonsillar ring. The lesion measured $5.0 \times 3.0 \times 2.0 \mathrm{~cm}$, it had a soft, rubbery consistency, and a fairly uniform red-purple colouration. There was no ulceration. Immunohistochemical staining revealed that the malignant lymphocytes expressed CD20, CD5, CD43, Bcl-2,Bcl_1, but not CD10 or CD23 antigens.

Extra-nodal lymphomas are frequently not diagnosed because their symptoms are similar to many other diseases of inflammatory etiology. During differential diagnostics procedure all diseases which are characterized by 
lymphadenopathy, such as toxoplasmosis, mononucleosis, lymphadenitis, metastases and Hodgkin's disease, must be considered [2]. In the oral localisation of lymphoma, clinical lesions resemble gingivitis, epulis, vascular angio granuloma, granuloma of pregnancy, drug-induced hypertrophy, tuberculosis, or lesions in AIDS. Proper treatment depends on the histological type and progression of the tumour. In extra-nodal lymphoma, surgical resection of the lesion and radiotherapy are used [2]. In aggressive lymphomas, combined local treatment with complementary or inductive radiotherapy or chemotherapy are applied [15, 16]. Similar clinical and radiological signs and symptoms, as well as results of laboratory tests in the case of head and neck lymphomas and inflammations, may result in late diagnosis which significantly deteriorates prognosis in the severe diseases.

\section{CONCLUSIONS}

1. In differentiation of pathological lesions on the head and neck, lymphomas should be considered.

2. Prior to prosthetic procedures, patients should be examined clinically and radiologically in order to exclude pathology of maxilla and maxillary sinuses.

3. With the lack of therapeutic progress in the treatment of inflammation of the oral cavity mucosa which last longer than 2 weeks, an in-depth general diagnostics should be applied.

4. Thorough multidisciplinary diagnosis of oral cavity mucosa diseases aids early diagnosis and rapid treatment of some proliferative disorders which manifest in the facial skeleton (such as lymphomas).

\section{REFERENCES}

1. Landis S, Murray T, Bolden S. Canser Statistics 1999. Canser J. Clin. 1999, 49, 28-31.

2. Lewandowski L, Nowaczyk MT. Non-Hodgkin's Lymphomas oral Cavity and Neck-diagnosis, treatment. Poznańska Stom. 2002, 24, 15-18.

3. Frei M, Dubach P, Reichart PA, Schmitt AM, Muller-Garamvolgyi E, Bornstein MM. diffuse swelling of the buccal mucosa and palate as first and only manifestation of an extranodal non-Hodgkin, double-hit, lymphoma; report of a case. Oral Maxillofac. Surg, 2012, 16, 69-74.

4. Chloupek A, Sarosiek T, et al. Malignant B-cell lymphoma. A case report of a malignant lymphoma of high grade malignancy located in the oral cavity. Cas. Stomat. 2003; 56, 12, 857-864.

5. Nadendla KL, Meduri V, Paramkusam G. Imaging characteristics of diffuse large cell extranodal non-Hodgkin's lymphoma involving the palate and maxillary sinus: a case report. Imaging Science in Dentistry. 2012, 42, 111-114.

6. Epstein JB, Epstein JD, Le ND, Gorsky M. Characteristics of oral and paraoral malignant lymphoma: a population -based review of 361 cases. Oral Surg Oral Med Oral Pathol Oral Radiol Endos.2001;92:519-525.

7. Jaffe ES et al.(2001) In Kleihues, P.Sobin, L.(eds) WHO classification of Tumours.ARC Press, Lyon.

8. Gerber-Leszczyszyn H, Łuczak k, Ćwioro F. Head and Neck nonHodgkin lymphoma. Czas stom.1993, 46: 115-118.

9. Etemad-Moghadam's, Tirgary F, Kehavarz S, Alaeddini M. Head and neck non-Hodgkin's lymphoma: a 20-year demographic study of 381 cases. Int. J. Oral Maillofac. Surg 2010, 39, 869-872.

10. Sugimoto KJ et al.Primary gingival diffuse large B-cell lymphoma: a case report and a review of the literature. Int J Clin Exp Pathol.2014; 7(1): 418-424.

11. Rosenwald A et al.The use of molecular profilling to predict survival after chemotherapy for diffuse large B-cell lymphoma. N Engl J Med. 2002.346: 1937-1947.

12. Sato Y, Onishi N, Morito T, Takata K, Mizobuchi K, Nagatsuka H, Ichimura K, Tanaka T, Tamura M, Yoshino T.Patients with localized primary non-tonsillar oral diffuse large B-cell lymphoma exhibit favorable prognosis despite a non-germinal center B-cell -like phenotype.Cancer Sci.2009; 100: 42-46.

13. Bhattacharyya I, Chehal HK, Cohen DM, et al. Primary diffuse large B-cell lymphoma of the oral cavity: germinal center classification. Head and Neck Pathol. 2010; 4, 181-191.

14. Chung-Che Chang, Rowe JJ, Hawkins P. Mantle cell lymphoma of the hard palate: A case report and review of the differential diagnosis based on the histomorphology and immunophenotyping pattern. Med. Oral Pathol. Oral Radiol. Endod. 2003, 96, 316-320.

15. Janas A, Grzesiak-Janas G. Non-Hodgkin extranodular lymphona of the palate. Pol.Merc.Lek.2006, 20, 120, 705-707.

16. Guggisberg K, Jordan RCK. Mantle cell lymphoma of the oral cavity: case series and comprehensive review of the literature. Oral Surg. Oral Med. Oral. Path. Oral Rad. and Endodont. 2010, 109, 1, 98-104. 